\title{
XXV. Ueber einige krystallographische Constanten des Korund.
}

\author{
Von
}

G. Melczer in Budapest.

(Hierzu Taf. XIV und XV.)

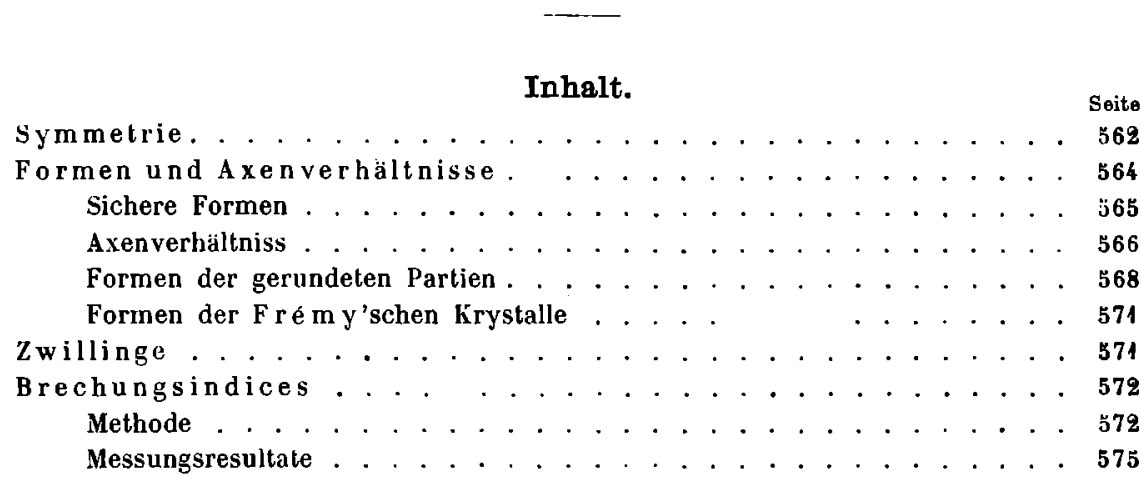

Vorliegende Untersuchungen beziehen sich auf eine Suite von mehr als 150 birmaner Rubinkrystallen, welche ich theils IHerrn Prof. Groth, theils Herrn A. Hahn in Idar zu verdanken habe, ferner auf drei ceyloner Korundkrystalle, welche mir ebenfalls Herr Prof. Groth zur Untersuchung überliess (zwei von diesen hat Herr Dr. F. Grünling, kgl. Custos, gesammelt), endlich auf eine Suite künstlicher Frémy'scher Rubinkrystalle, welche mir auf meine Bitle Herr Prof. A. Lacroix zur Verfügung stellte. Es sei mir erlaubt, den genannten IJerren für ihre Iiebenswürdigkeit auch an dieser Stelle meinen besten Dank auszusprechen, sowie auch Herrn Prof. Krenner, mit dessen Erlaubniss ich diese, im mineralogischen Institute der Münchener Universität begonnenen Studien im mineralogischen Institute der Budapester Universität fortsetzen und beendigen konnte.

Der Zweck meiner Untersuchungen war die Ergänzung unserer einigermassen lückenhaften Kenntniss über dieses Mineral und zwar betreffend seine Symmetrie, sein Axenverhältniss und scine Hauptbrechungsexponenten. 


\section{Symmetrie.}

Der Korund wurde von jeher für ein trigonal-skalenoëdrisches Mineral angesehen, jedoch Mallard und Tschermak haben auf Grund seines Verhaltens im convergenten polarisirten Lichte diese seine Symmetrie bezweifelt, weil nämlich viele Korundkryslalle ein den optisch \%weiaxigen Substanzen : ihnliches Axenbild liefern.

Mallard") hält ihn deshalb für ein aus optisch zweiaxigen Lamellen gebildetes pseudohexagonales Mineral; Tschermak ebenso ${ }^{2}$ ), und zwar möglicherweise für monoklin, indem er darauf hinweist, dass von den lhomboëderflïchen oft eine herrscht und dass die Zwillingslamellen oft nur nach zwei lhomboëderflächen vorhanden sind, demzufolge auch dic Streifung auf der Basisfläche einen monoklinen Charakter hat. Unter den übrigen Forschern hat sich mit der Symmetrie besonders Lasaulx beschäftigt ${ }^{3}$ ). Er hat von verschiedenen Fundorten stammende Korunde untersucht und gefunden, dass die optische Zweiaxigkeit dieses Minerals mit dem Vorhandensein der Zwillingslamellen nach den Flächen des Grundrhomboëders und mit der schaligen Zusammensetzung nach den Prismenflïchen im Zusammenhange steht, indem solche Krystalle, an welchen (obiges nicht vorhanden ist, ein ungestörtes einaxiges Bild liefern.

Ich kann die Beobachtungen Lasaulx's durch meine Erfahrungen an den birmaner Krystallen nur bestïtigen. Ich untersuchte diesbezüglich etwa 50 Krystalle; unter diesen gaben nur sechs ein deutlich zweiaxiges Bild, und fünf von dicsen sechs Krystallen zeiglen sehr deutlich ein oder mehrere Zwillingslamellen nach ein oder auch zwei Rhomboëderflächen. Verdeckt man diese Theile der Krystalle, so sieht man das ungestörte einaxige Bild, so wic bei denjenigen Krystallen, in welchen keine Zwillingslamelle ist. Dass aber auch diese zweiaxigen Bilder nicht normale sind, kann man bei der Drehung des Krystalles nm $45^{\circ}$ sehen; es ist dann nämlich nur der innerste, bis zu den ersten Lemniscaten reichende Theil der Ilyperbeln sichtbar $\left.{ }^{4}\right)$. Schaut man durch solche Partien, welche Zwillingslamellen nach $z$ wei Rhomboëderflächen enthalten, so ist das Axenbild ganz gestört.

In den Frómy'schen Krystallen sind keine Zwillingslamellen zu bemerken, und sie geben auch sümmtlich ein ungestörtes einaxiges Bild.

Schon dieses optische Verhalten zeigt zur Genüge, dass der Korund optisch einaxig, also - mit Ilinzuziehung seiner geometrischen Ausbildung

1) Ann. des mines 1876 (X). Ref. diese Zeitschr. 1877, 1, 349.

2) Min. u. petr. Mittheil. Neue Folge. 1878, 1, 362. Ref. diese Zeitschr. 4, 224.

3) Diese Zeitschr. 1883, 10, 346-365.

i) Genau dasselbe sieht man mit dem Polarisationsmikroskop an dem basischen Schliffe eines Calcitkrystalles, welcher einige $Z$ willingslamellen enthält. 
- trigonal-skalenoëdrisch sei. Um diese Annahme auch geometrisch zu bekräftigen, habe ich an einigen recht gut ausgebildeten Krystallen in allen drei Zonen die Winkel or resp. ea, $r$ gemessen ${ }^{1}$ ). (Als Instrument diente bei diesen, sowie bei nachfolgenden Messungen theils ein Fuess'sches grosses Reflexionsgoniometer (Modell Nr. l), theils ein gewöhnliches ReIlexionsgoniometer (Modell Vir. II) derselben Firma. Ich habe vor Beginn der Messungen beide in der gewohnten Art rectificirt.) Das Resultat ist folgendes:

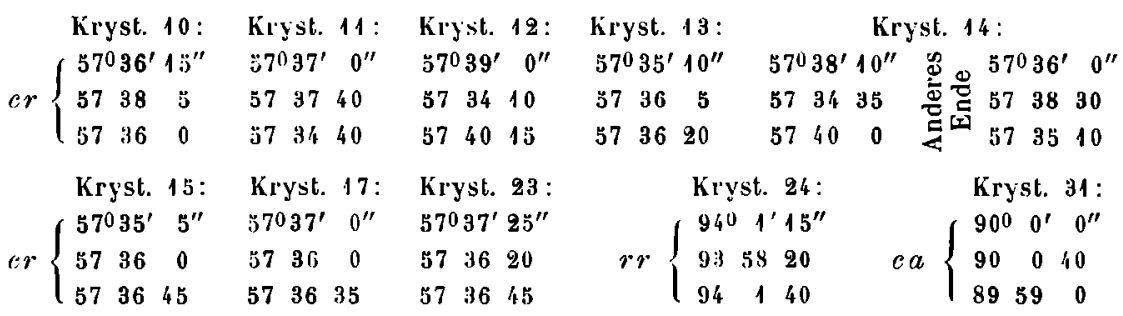

Den Abweichungen ist also keinerlei Regelmässigkeit eigen. Im Einklange mit diesem Resultate slehen auch alle übrigen Messungen, indem sich nümlich für den Fall, dass Korund monoklin wäre, die gemessenen Werthe für ar (Zone arr), en und eventucll auch für or um zwei Mittelwerthe gruppiren müssten, was aber keineswegs der Fall ist.

Es ist also nach Obigem unzweifelhaft, dass Korund so wie ehemals als trigonal zu betrachten ist.

Was nun den Grad der Symmetrie innerhalb dieses Systems betrift, so zeugen die Ausbildung der Formen, dic Abwesenheit tetartoëdrischer Formen und die an einigen Krystallen beobachteten natürlichen Aetzfiguren für die skalenoëdrische Abtheilung. Nur ein Krystall ist scheinbar hemimorph entwickelt, indem an ihm $r$ und $n$ an dem einen Ende mit grossen, an dem anderen mit auffallend kleinen Flächen entwickelt sind. Die pyroëlektrische Untersuchung desselben nach der Methode Kundt's, welche Herr V. v. Worobieff - gerade mit ähnlichen Untersuchungen an Turmalinen beschäftigt - auf meine Bitte vornahm und mehrfach wiederholte, zeigte jedoch keine Polarität der Hauptaxe. Dementsprechend sind auch die natürlichen Aetzfiguren, wo sie auf beiden Basisflächen vorhanden sind, gleich.

Die Aetzfiguren $\left.{ }^{2}\right)$ bestehen auf den Basisflächen aus dreiseitigen kleinen Vertiefungen (Fig. 18, Taf. XV), mit meist ein wenig gekrümmten Flächen.

1) Bezüglich der Buchstabenbezeichnung der Formen habe ich mich an die neueste (6.) Ausgabe von Dana's System (1892) gehalten. Siehe auch S. 565.

2) Für solche muss man sie wohl halten und nicht etwa für Folgen gestörter Flächenausbildung, da sie an mehreren Krystallen ganz gleichmüssig ausgebildet vorkommen. 
.luf einigen Krystallen sind jedoch ihre Flächen ganz scharf ausgebildet und an diesen sieht man mit dem Goniometer, dass sie genau in die Zonen or fallen, also Thomboëderflächen sind. Ihre Neigung konnte an einern Krystalle auch gemessen werden. Diese Messung deutet annähernd auf $\{4.33\}=\{1.0 . \overline{1} .10\}:$

$$
(111):(433)=(0001):(1.0 . \bar{T} .10)=9^{0} 12^{\prime} \quad 8^{0} 57^{\prime} 30^{\prime \prime}
$$

Auf den Prismenflächen sind die Aetzfiguren rhomboïdische Vertiefungen (Fig. 19, Taf. XV): Combinationen von Pyramiden zweiter Art mil cin oder mehr Skalenoëdern. Auऍ zwei benachbarten Prismenflächen liegen die Aetzfiguren immer symmetrisch zu einander. Nach Bauer ${ }^{1}$ ) ist dasselbe auch für die auf den $n$-Fllächen befindlichen Aetzfiguren der Fall.

Iie auf den Flächen des Grundrhomboëders oft vorkommende Streifung in der Zone $[r n]$ kann vielleichl auch als Aetzerscheinung aufgefasst werden; denn wäre sic cine Combinationsstreifung durch Skalenoëder dieser Zone, so würde sie auf der ganzen Fläche vorhanden sein, sie kommt jedoch meist nur in der Nähe der Basisflüche vor.

Auch die Fortwachsungsflächen, welche man auf den Basisflächen gewöhnlich bemerken kann, widersprechen der trigonal-skalenoëdrischen Symmetrie nicht. Meist ist eine feine trigonale Streifung parallel den Kanten $\mathrm{cr}$ zu bemerken, wie auch an Korunden anderen Vorkommens. An solchen Krystallen, an welchen dicse Streifung gröher ist, kann man sehen, dass sie durch Alternation der Basisflïche mit schmalen IIächen des Grundrhomboëders bedingt ist. An vielen Krystallen jedoch nehmen, wie man mit dem Goniometer aus den Reflexzügen sieht, auch Flächen aus den Zonen $[c n]$ und $[c s]$ an dieser Streifung Theil (Fig. 20 und 21), und wenn dann von den alternirenden Flächen einige fehlen, dann sind diese Fortwachsthumsfiguren scheinbar asymmetrisch resp. monosymmetrisch, was Tschermak - wie schon erwähnt - bowog, auf dic monokline Symmetrie des Korundes zu schliessen.

An der erwähnten Streifung, welche jedoch oft so fein ist, dass sie die Reflexion gar nicht beeinträchtigt, kann man die Basisllächen, im Falle die Krystalle verzerrt sind, immer gut erkennen, ebenso die Rhomboëderflächen an der erwähnten Streifung.

Schliesslich bemerke ich noch, dass auch die zwischen den Zonen der ebenen Flächen vorhandene Rundung der trigonal-skalenoëdrischen Symmetrie entspricht.

\section{Formen und Axenverhältnisse.}

Die von mir untersuchten birmaner Krystalle sind sämmtlich einzelne Krystalle, durchschnittlich hirse- bis erbsengross und mit wenig Ausnahmen

1) l. c. S. 215 . 
lichtrolh, mit sehr wenig bläulicher Farbennüance. Bezüglich ihres Ilerkommens konnte mir IIerr $\Lambda$. Hahn nur soviel mittheilen, dass sie aus den (irulen der Burma Ruby Mining Company stammen. Dia sie Jlabitus, (riösse und Farbe anbelangend ganz den von Bauer ${ }^{1}$ beschriebenen Krystallen ähnlich sind, so stammen sie wahrscheinlich auch aus dem krystallinischen Kalke der lhubingegend von Ober-Birma, deren Mittelpunkt Mogouk ist.

Hauptformen der ron mir untersuchten birmaner Krystalle sind folgende sehr gewöhnliche Formen:

$$
\begin{aligned}
& c\{111\}=\{0001\} 0 R \\
& r\{100\}=\{10 \bar{T}\} R \\
& n\{31 T\}=\{22 \pi 3\} T^{P 2} \\
& a\{10 \bar{T}\}=\{11 \overline{2} 0\} \infty P^{2} .
\end{aligned}
$$

Ausser diesen kommen mit gut entwickelten Flächen nur folgende drei vor, von denen die ersten zwei ebenfalls häufige formen des Rubins sind:

$$
\begin{aligned}
w\{41 \overline{2}\} & =\{11 \overline{2} 1\} 2 P 2 \\
v\{51 \overline{3}\} & =\{44 \overline{8} 3\} \frac{8}{3} P^{2} \\
\{41 \overline{1}\} & \left.=\{50 \overline{5} 2\} \frac{5}{2} R^{2}\right)
\end{aligned}
$$

und zwar die erste als herrschende Form an einem Krystalle (Fig. 17), die letzteren zwei an je cinem Krystille mit zwar nur je einer, aber gut ausgebildeten Flïche.

Hïufig sind also nur $c, r, n$ und $a$. Die verschiedene quantitative $\Lambda$ usbildung dieser Formen bedingt jedoch einen sehr wechselvollen llabitus; dic diesbezüglichen Typen und einzelne Uebergänge habe ich in 'Taf. XIV und XV, Fig. 1-17 mit eincm Drehungswinkel von $20^{\circ}$ und einem Elevationswinkel von $10^{\circ}$ construirt. Wie aus den Figuren zu ersehen, kann man lafelförmige, kurzprismatische, prismalischc, oktaëderähnliche (wenn $c$ und $r$ gleich gross ausgebildet), rhomboëdrische, tonnenlörmige und bipyramidale Typen unterscheiden. Am häufigsten sind die in Fig. 7. 9 and 11 gezeichneten Combinationen, am seltensten Fig. 1\%, 16 und 17. (Ein Theil dieser Combinationen ist auch in Bauer's Arbeit über den Rubin von Birma 3) gezeichnet, jedoch, wie man sofort bemerken und sich durch Construction auch überzeugen kann, mit cinem falschen Axenkreuz.)

Schr häulig kommen auch durch Vorherrschen von zwei oder vier

1) I. c. S. 20 is.

2) Dieses Rhomboeder ist weder in Dana's Handbuch, noch in Goldschmidt's Index und Winkeltabellen enthalten. Striuver führt für korund nicht $\{4 \bar{T}\}$ an (Ref. N. Jahrb. f. Min. 1872, 425), sondern $\{411\}=\{04 \bar{\jmath}\}$ (Ematite di Traversella 1872, S. 14 $\}$. Die Form ist also für Korund neu.

3! !. c. Taf. VII. 
Rhomboëderflächen oder von zwei Prismenflächen tafelfürmig resp. prismatisch verzerrte Krystalle vor.

Das Axenverhältniss des Korund ist aus den Grundwerthen Miller's $\left.{ }^{1}\right)$ (und zwar aus $e r, r r$ und $e n$ j berechnet $1: 1,3630$. Dieses Axenverhältniss wurde von den meisten Forschern, die sich mit Korund beschäftigten, namentlich Kokscharow ${ }^{2}$ ), Klein ${ }^{3}$ ), Des Cloizeaux ${ }^{4}$ ), Bauer ${ }^{5}$ ), Busz(i), acceptirt. Jeremejew hat auf Grund seiner an turkestanischen Krystallen angestellten Messungen das etwas abweichende Verhältniss 1:1,3636 abgeleitet ${ }^{7}$ ), und letzteres nimmt Goldschmidt in seinen Winkeltabellen an. Ich habe leider keine Kenntniss davon, wie viel Krystalle Jeremejew zu diesem Zwecke gemessen hat und wie dieselben ausgebildet sind, da mir nur das Referat seiner Arbeit zur Verfügung stand, jedoch dürften dieselben laut seiner Winkeltabelle schwerlich so vollkommen ausgebildet sein, wie meine birmaner Krystalle. Aus diesem Grunde, und weil die Winkelwerthe der übrigen erwähnten Forscher nur formenbestimmonden Werth haben, da sie sich auf je ein bis zwei, und theilweise nicht gut ausgebildete Krystalle beziehen, fand ich es für zweckmässig, das Axenverhältniss des Korundes neu zu bestimmen, was auch Dank dem neuen vorzüglichen birmaner Material gut gelang. $\mathrm{Zu}$ diesem $\mathrm{Zwecke}$ dienten in erster Linie die Werthe von $c r$, sodann $a r$ und $r r$, und aus diesen berechnet sich das Axenverhältniss zu $1: \mathbf{1 , 3 6 5 2}$ oder nach Miller $a=85^{\circ} 422^{\prime}$. Die Uebereinstimmung zwischen den gemessenen und berechneten Werthen ist wegen der vorzüglichen Reflexion der Flächen eine sehr befriedigende. (In der Tabelle bedeutet $\mathbf{K r}$. die Zahl der gemessenen Krystalle und $n$ die der Kanten, $\pm d$ ist die mittlere Differenz zwischen den gemessenen Werthen und dem aus ihnen gebildeten Mittelwerthe, d. h. das arithmetische Mittel dieser Differenzen. Aus der so gebildeten Mitteldifferenz kann man besser beurtheilen, inwiefern die gemessenen Werthe sicher sind, als durch Angeben der Grenzwerthe.)

$$
\begin{aligned}
& \text { Gemessen Kr. } n \quad \pm d \text { Berechnet: } \\
& c r=(111):(100)=(0001):(10 T 1)=57036 \frac{1}{3}^{\prime} \quad 16 \quad 50 \quad \frac{3^{\prime}}{4} \quad 57036^{\prime} 38^{\prime \prime} \\
& a r=(10 \pi):(100)=(1+\overline{2} 0):(10 T 1)=42 \quad 59 \frac{1}{2} \quad 8 \quad 21 \quad \frac{3}{4} \quad 43 \quad 0017 \\
& r r=(010):(100)=(10 \pi 1):(1101)=94 \quad 0 \frac{1}{2} \quad 6 \quad 9 \quad 1 \frac{1}{2} \quad 935925 \\
& c n=(111):(31 T)=(0001):(22 \overline{1} 3)=61 \quad 12 \frac{1}{4} \quad 5 \quad 3 \quad 7 \quad 1 \frac{1}{2} \quad 61 \quad 13 \quad 1 \\
& r n=(100):(31 T)=(10 \bar{T}):(22 \overline{4} 3)=25 \quad 59 \frac{3}{4} \quad 3 \quad 3 \quad 8 \quad \frac{1}{2} \quad 25 \quad 5926
\end{aligned}
$$

1) Mineralogy 1852, S. 243.

2) Mat. Min. Russl. 1, 23 und 6, z23.

3) N. Jahrb. f. Min. etc. $1871,486$.

4) Compt. rend. 1888, 106, 567. Hef. diese Zeitschr. 18, 323.

5) 1. c. 212.

6) Diese Zeitschr. 15, 622.

7) Ref. diese Zeitschr. 4, 642. 


$$
\begin{aligned}
& \text { Gemessen: Kr.: } n \pm d \text { Berechnet: } \\
& c w=(111):(41 \overline{2})=(0001):(11 \overline{2} 1)=69^{0} 32 \frac{3}{4}^{\prime} \quad 1 \quad 4 \quad 1 \frac{1}{4}^{\prime} 69^{0} 53^{\prime} 6^{\prime \prime} \\
& c v=(111):(51 \overline{3})=(0001):(44 \overline{8} 3)=74391 \quad 1 \quad 1-743826 \\
& (111):(4 T T)=(0001):(5052)=7546 \frac{1}{4} \quad 1 \quad 1-754544
\end{aligned}
$$

Hier theile ich ferner die an einem schönen blauen ceyloner Krystalle und die an Frémy'schen künstlichen Krystallen ausgeführten Messungen mit.

Dieser ceyloner Krystall (im Besitze der k. bayerischen Mineraliensammlung des Staates) ist beinahe erbsengross, hellblau und zur Hälfte ausgebildet. Seine herrschende Form. ist $n\{31 T\}=\{22 \overline{4} 3\}$, deren Kanten schmale Flächen von $r\{100\}=\{10 T 1\}$ abstumpfen. Seine Werthe:

$$
\begin{aligned}
& \text { Gemessen: } n \pm d \text { Berechnet: } \\
& \left.e n^{1}\right)=(111):(31 T)=(0001):(22 \overline{4} 3)=61012 !^{\prime} \quad 2 \quad 0^{\prime} \quad 61013^{\prime} 1^{\prime \prime} \\
& r n=(100):(31 T)=(10 T 1):(22 \overline{4} 3)=25 \quad 39 \frac{1}{2} \quad 6 \quad 1 \frac{3}{4} \quad 25 \quad 5926
\end{aligned}
$$

Die Frémy'schen Krystalle sind mit Ausnahme einiger grüsserer Täfelchen $1-2 \mathrm{~mm}^{2}$ gross und von der in Fig. 2 wiedergegebenen Ausbildung, meist aber noch dünner. Ausser der Basis und den Rhomboëderflächen ist an ihnen gewöhnlich auch $n\{31 \bar{T}\}=\{22 \overline{1} 3\}$ mit winzigen Flächen vertreten und an einem Täfelchen (Fig. 22) ist auch noch eine Fläche von $\eta\{22 T\}=\{01 T 1\}$ vorhanden. Sie sind etwas dunkler gefärbt als die erwähnten birmaner Krystalle und haben einen deutlicheren Stich ins Violette. Mit diesen künsllichen Rubinkrystallen hat sich bereits Des Cloizeaux beschäftigt und sie kurz beschrieben ${ }^{2}$ ), jedoch können seine Messungen für die Beurtheilung des Axenverhältnisses nicht massgebend sein; er sagt nämlich, dass die Krystalle nur scheinbar vollkommen entwickelt sind und keine guten Reflexe geben. Ich kann dies von den Krystallen, welche mir durch die Güte des Herrn Prof. Lacroix zur Verfügung standen, nicht behaupten, denn unter ihnen giebt es genug solche, welche ideale Reflexe liefern.

Die gemessenen und berechneten Winkel dieser Krystalle sind ${ }^{3}$ ):

$$
\begin{aligned}
& \text { Gemessen: Kr. } n \pm d \text { Berechnet: } \\
& \text { cr }=(111):(100)=(0001):(10 \overline{1})=570362^{\prime} \quad 11 \quad 44 \quad \frac{f^{\prime}}{2} \quad 57^{\circ} 36^{\prime} 39^{\prime \prime} \\
& c n=(111):(31 T)=(0001):(22 \overline{4} 3)=61 \quad 13 \frac{1}{3} \quad 3 \quad 14 \quad 1 \frac{1}{2} \quad 61 \quad 13 \quad 1 \\
& r n=(100):(31 T)=(10 \bar{T}):(2243)=2559 \frac{1}{4} \quad 1 \quad 1-255926 \\
& c \eta=(111):(221)=(0001):(01 T 1)=5739 \frac{1}{3} \quad 1 \quad 1 \quad-\quad 373639
\end{aligned}
$$

Aus diesen und den vorhergehenden Winkelwerthen ist evident, dass das berechnete Axenverhältniss $1: \mathbf{1 , 3 6 5 2}$, welches laut den gemessenen

$$
\begin{array}{ll}
\text { 1) } \operatorname{resp.} \frac{n n}{2} \cdot & \text { 2) l. c. }
\end{array}
$$

3) Ich kann njcht umhin, an dieser Stelle zu erwähnen, dass ich bei der Messung der kleinen Frémy'schen krystalle mit Vortheil den Goldschmidt'schen kleinen Justirapparat (diese Zeitschr. 1892, 20, 344) benutzte, indem ich mich von der Stabilitát und Brauchbarkeit dieses kleinen Attributes für Goniometer Nr. II überzeugte. 
Winkeln bis auf $\pm 0,0001$ sicher ist, für den Korund im Allgemeinen gültig ist.

Formen der gerundeten Partien. Die birmaner Krystalle sind zwischen den Flächen der aufgezählten Formen, also in den Zonen [ena] $[n r n][c r][a r]$, sowie in der Gegend der negativen Rhomboëder melır oder weniger gerundet, wie geflossen. Diese gerundeten Partien kann man woll nicht als Folgen nachtrüglicher Lösung betrachten, denn die Flächen der Ilauptformen sind beinahe immer glatt, spicgeln vortrefflich, und Aetzfiguren kommen auf ihnen selten vor und auch dann sind sie klein und scharf. Ich halte vielmehr diese gerundelen Partien für ursprünglich, da die Krystalle - wie schon erwähnt - wahrscheinlich in Kalken durch Contactmetamorphose entstanden sind, wie die von Bauer beschriebenen birmaner Krystalle. Diese gerundeten Partien können also als Uebergangsflächen im Sinne Goldschmidt's bezeichnet werden.

Auf dem Goniometer liefern diese gerundeten Partien Reflexzüge, welche jedoch nicht so ununterbrochen sind, dass sie durch Interferenz zu erklären wären, sondern die einzelnen Reflexe in ihnen sind spärlicher und einige davon sind relativ lichtstark, so dass sie mit einer Sicherheit von 2-10' einzustellen waren, ihnen entsprechende gut begrenzte Flächenpartien konnte ich jedoch in den meisten Fällen nicht entdecken. Ich habe diese Reflexe an acht Krystallen - in einigen auch mit verschiedener Incidenz - eingestellt und dadurch resultirte eine ganze lieihe von Bipyramiden zweiter Ordnung, ferner (theils von diesen, theils von anderen ähnlichen Krystallen) mehrere positive Rhomboëder und Skalcnoëder und cin dihexagonales Prisma, welche sümmtlich in folgender Tabelle enthalten sind. In den negativen Sextanten habe ich blos die zwci einfachen Rhomboëder $\{110\}$ $=\{01 \overline{1} 2\}$ und $\{11 \overline{1}\}=\{02 \overline{2} 1\}$ gefunden, welche vom Korund bereits bekannt sind. Ausser diesen letzteren werden die gerundeten Partien hier noch von mehreren negativen Skalenö̈dern gebildet, aber diese fallen nicht in die Hauptzonen, und wäre ihre Bestimmung demnach mit einkreisiger Messung allzu schwierig gewesen.

Messungen an den gerundeten Partien der birmaner Krystalle.

Negative Rhomboëder.

$$
\begin{aligned}
& \bullet s=(1+1):(11 \overline{1}) \quad=(0001):(02 \overline{2} 1)=72^{0} 23^{\prime} 574^{\prime} 72024^{\prime} 7^{\prime \prime}
\end{aligned}
$$

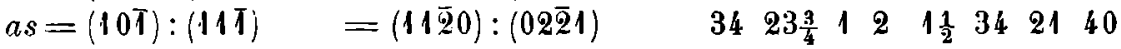

$$
\begin{aligned}
& (111):(110)=(0001):\left(01 \text { T2) } \quad 38 \quad 24 \frac{1}{2} 12 \quad \frac{1}{4} 38 \quad 14 \quad 42\right.
\end{aligned}
$$

Positive Rhomboëder:

$$
\begin{array}{rlrrrrrrrrr}
(111):(16.13 .13) & =(0001):(1.0 . \overline{1} .14) & 6 & 30 & 1 & 1 & - & 6 & 25 & 28 \\
:(433) & = & :(1.0 . \overline{1} .10) & 8 & 55 & 1 & 2 & 1 & 8 & 57 & 30 \\
:(755) & = & :(2.0 .2 .17) & 10 & 31 \frac{1}{4} & 1 & 1 & -10 & 30 & 24
\end{array}
$$




$$
\begin{aligned}
& (111):(10.7 .7)=(0001):\left(10^{\overline{1}} 8\right)=10^{\circ} 58^{\prime} 11-11^{\prime} 8^{\prime} 50^{\prime \prime} \\
& :(22.13 .13)=\quad:(3.0 . \overline{3} .16) \quad 1622 \quad 11-162759 \\
& :(13.1 .1)=:(40.5) \quad 5132 \quad 11-513515 \\
& :(16.1 .1)=:(50 \overline{5} 6) \quad 523111-524314 \\
& :(22.1 .1)=:(70 \overline{7} 8) \quad 3358 \frac{1}{2} 1211^{\prime} 54 \quad 331 \\
& :(38 . \bar{T} . \bar{T})=:(13.0 . \bar{T} \overline{3} .12) \quad 59421 \quad 11-593854 \\
& :(43 . \overline{2} . \overline{2})=:(15.0 . \overline{5} .13) 61121.11-611156 \\
& :(17 . \overline{1} . \overline{1})=:(60 \overline{6} 5) \quad 62 \quad 3 \frac{1}{2} 11-62459 \\
& :(43 . \overline{5} . \overline{5})=:(16.0 . \overline{6} 6.11) 6623 \frac{1}{2} 11-662613 \\
& :(13 . \overline{2} . \overline{2})=:(5053) \quad 6853 \quad 11-69428
\end{aligned}
$$

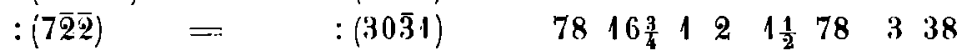

Bipyramiden zweiter Art.

$$
\begin{aligned}
& (111):(22.7 . \overline{8})=(0001):(5.5 . \overline{1} \overline{0} .7) \quad 62 \quad 47 \frac{1}{4} 33 \quad 3 \quad 5 \quad 6251 \quad 13
\end{aligned}
$$

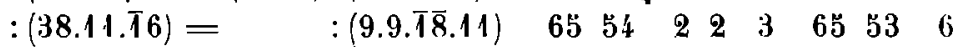

$$
\begin{aligned}
& :(11.3 . \overline{5})=:(8.8 . \overline{1} \overline{6} .9) \quad 67 \quad 31 \quad 2 \quad 5 \quad 4 \quad 67 \quad 36 \quad 23 \\
& :(15.4 . \overline{7})=:(11.11 . \overline{2} \overline{2} .12) 68 \quad 6 \frac{1}{2} 222 \quad 3 \quad 68 \quad 13 \quad 16 \\
& :(17.4 . \overline{9})=:(13.13 . \overline{2} \overline{6} .12) 711611-711915
\end{aligned}
$$

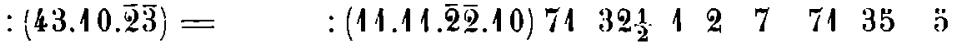

$$
\begin{aligned}
& :(9.2 . \overline{5})=\quad:(7.7 .17 .6) \quad 72 \quad 25 \quad 2315 \quad 72 \quad 34 \quad 18
\end{aligned}
$$

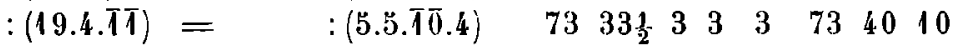

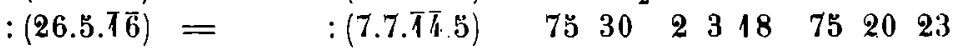

$$
\begin{aligned}
& :(11.2 \overline{7})=:(33 \overline{6} 2) \quad 76 \quad 21 \quad 1 \quad 314 \quad 76 \quad 16 \quad 44
\end{aligned}
$$

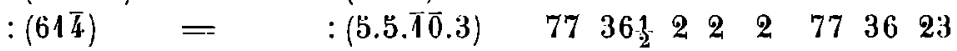

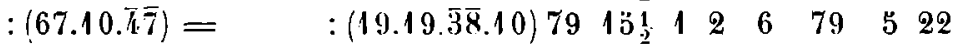

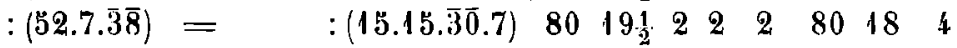

$$
\begin{aligned}
& :(17.2 . \overline{3})=\quad:(5.5 . \overline{1} \overline{0} .2) \quad 8131 \quad 2 \quad 2 \quad 9 \quad 8139 \quad 56
\end{aligned}
$$

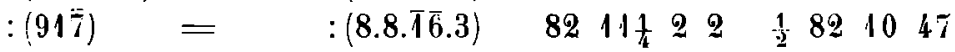

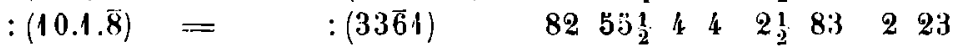

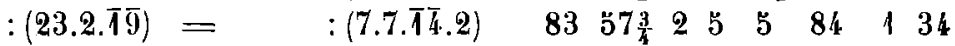

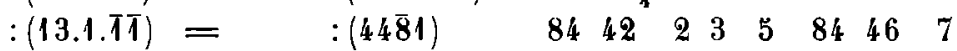

$$
\begin{aligned}
& :(29.2 . \overline{2} \overline{5})=\quad:(9.9 . \overline{1} \overline{8} .2) \quad 85 \quad 13 \quad 2 \quad 3 \quad 6 \quad 8520 \quad 50 \\
& :(15.1 . \overline{3})=\quad:(14.14 . \overline{2} \overline{8} .3) 8530 \frac{1}{2} 11-853045 \\
& :(35.2 . \overline{3} \bar{T})=\quad:(11.11 . \overline{2} \overline{2} .2) \quad 86 \quad 10 \frac{1}{2} 22 \begin{array}{llllll}
2 & 2 & 3 & 86 & 11 & 25
\end{array} \\
& \text { Zone }[10 \overline{1}: 100]=[11 \overline{2} 0: 10 \overline{1}] \\
& (10 \bar{T}):(11.0 . \overline{2})=(11 \overline{2} 0):(11.2 . \overline{3} .9)=2659 \frac{1}{4} 11-265824 \\
& \text { Zone }[01 T: 100]=[\text { T2T0: 10T0] } \\
& (01 \bar{\top}):(13.6 . \overline{6})=(\text { T2T0 }):(7.12 . \overline{9} .13) \quad 5552 \frac{1}{2} 11-555844 \\
& :(10.3 . \overline{3})=:(7.6 . \overline{3} .10) \quad 662511-661833 \\
& :(18.5 . \bar{S})=\quad:(13.10 . \overline{2} \overline{3} .18) 67521335 \% 6753 \text { \&1 }
\end{aligned}
$$




$$
\begin{aligned}
& (01 \overline{1}):(51 T)=(\bar{T} T \bar{T} 0):(42 \overline{6} 5)=73^{0} 50^{\prime} \quad 1211^{\prime} 73^{\prime} 41^{\prime} 41^{\prime \prime} \\
& :(17.3 . \overline{3})=:(14.6 . \overline{2} 0.17) 7533 \frac{3}{4} 11-753139 \\
& :(91 \overline{1})=:(8.2 . \overline{10} .9) \quad 8052 \quad 1 \quad 1-804.610
\end{aligned}
$$

Dihexagonales Prisma:

$$
(10 \bar{T}):(4 \overline{4} \overline{3})=(11 \overline{2} 0):(52 \overline{\bar{T}} 0)=1359 \frac{1}{2} 11-135352
$$

Diese Formen können natürlich für diese Krystalle nicht als sicher constatirt gelten. Es sind jedoch unter ihnen mehrere solche, welche von Korund schon bekannt sind, und zwar:

$$
\begin{aligned}
& s\{11 \overline{1}\}=\{02 \overline{2} 1\}-2 R \\
& \{110\}=\{01 \bar{T} 2\}-\frac{1}{2} R \\
& k\{92 \overline{5}\}=\{7.7 . \overline{1} 4.6\} \frac{7}{3} P 2 \\
& \left.\{19.4 . \bar{T} T\}=\{5.5 . \overline{0} .4\} \frac{5}{2} P 2^{1}\right) \\
& \theta\{91 \overline{7}\}=\{8.8 . \overline{1} \overline{6} .3\} \frac{16}{3} \mathrm{P2} \\
& v\{13.1 . \overline{1} \bar{T}\}=\{44 \overline{8} 1\} 8 P 2 \\
& w\{15.1 . \overline{1} \overline{3}\}=\{14.14 . \overline{2} \overline{8} .3\}{ }_{\overline{3}}^{28} P^{2} \\
& i\{41 \bar{T}\}=\{42 \overline{6} 5\} \frac{2}{5} R 3 .
\end{aligned}
$$

Diese sind also bis zu einem gewissen Grade wahrscheinlich. Ebenso können ihrer einfachen Indices und der Uebereinstimmung der gemessenen und berechneten Winkel wegen folgende als wahrscheinlich bezeichnet werden:

$$
\begin{aligned}
& \{433\}=\{1.0 . \overline{1} .10\}_{10}^{1} R \\
& \{13.1 .1\}=\{40 \overline{4} 5\} \frac{4}{5} R \\
& \{22.1 .1\}=\{70 \overline{7} 8\} \frac{7}{8} R \\
& \{17 . \bar{T} . \bar{T}\}=\{60 \overline{6} 5\} \frac{6}{5} R \\
& \{22.7 . \overline{8}\}=\{5.5 . \overline{10} . \overline{7}\}{ }^{10} \mathrm{P2} \\
& \{61 \overline{4}\}=\{5.5 . \overline{0} 0.3\} \frac{10}{3} P 2 \\
& \{10.1 . \overline{8}\}=\{33 \overline{6} 1\} 6 P 2 \\
& \{23.2 .1 \overline{9}\}=\{7.7 .14 .2\} 7 P 2 \\
& \{35.2 . \overline{3} T\}=\{11.11 . \overline{2} \overline{2} .2\} 11 P 2 \\
& \{91 \overline{1}\}=\{8.2 . \overline{0} 0.9\} \frac{2}{3} R \frac{5}{3} \\
& \{4 \overline{4} \overline{3}\}=\{52 \overline{7} 0\} \infty P_{\overline{5}}^{7}
\end{aligned}
$$

Die übrigen sind wenig wahrscheinlich und zum Theil vicinale Formen, nämlich :

$$
\begin{aligned}
& \left.\begin{array}{l}
\{13.0 . \overline{1} 3.12\} \\
\{15.0 . \bar{T} 5.13\}
\end{array}\right\} \text { vicinal } \mathrm{zu}\{100\}=\{10 \overline{1} 1\} \\
& \{16.0 . \overline{1} \overline{6} .11\} \text { vicinal zu }\{8 \overline{1}\}=\{30 \overline{3} 2\}
\end{aligned}
$$

1) Barvir, Ref. diese Zeitschr. 1896, 25, 431. 


$$
\begin{aligned}
& \left\{\begin{array}{l}
\{.8 . \overline{1} \overline{6} .9\} \\
\{11.11 . \overline{2} \overline{2} .12\} \\
\{13.13 . \overline{2} \overline{6} .12\} \\
\{11.11 . \overline{2} \overline{2} .10\}
\end{array}\right\} \text { vicinal zu }\{41 \overline{2}\}=\{11 \overline{2} 1\} \\
& \{19.19 . \overline{3} \overline{8} .10\} \\
& \{15.15 . \overline{3} \overline{0} .7\} \\
& \{7.12 . \overline{9} .13\} \text { vicinal zu }\{71 \overline{5}\}=\{22 \overline{4} 1\} \\
& \text { vicinal zu }\{21 T\}=\{12 \overline{3} 2\} .
\end{aligned}
$$

Im Allgemeinen kann man also sagen, dass diese gerundeten Partien der birmaner Krstalle theilweise von Flächenpartikelchen einfacher, wahrscheinlicher Formen gebildet werden.

Formen der Frémy'schen Krystalle. An den Frémy'schen Krystallen sind zwischen den Flächen der Hauptformen $(c, r, n)$ keine gerundeten Partien. An mehreren Krystallen scheint es, wie wenn die $c r$-Kanten durch sehr schmale Flächen abgestumpft wären. Mit dem Goniometer sieht man jedoch, dass diese nicht einheitlich sind, sondern aus unvollkommenen Streifen bestehen; nur an einem Krystalle war als derartige Abstumpfung eine gute liläche: $\{11.5 .30\}=\{20 \overline{2} 7\}$ vorhanden und an einem anderen Krystalle das Rhomboëder $\{13.13 .8\}=\{07 \overline{7} 6\}$ ebenfalls mit nur einer, aber guten. Flüche.

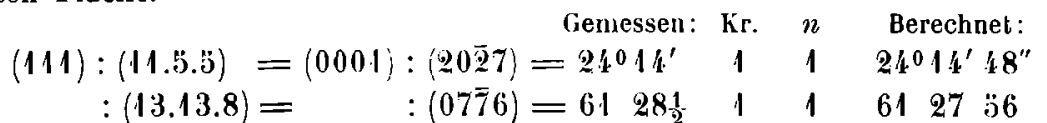

Diese zwei Formen kann man Obigem nach für sicher halten. Des Cloizeaux erwähnt von den Frémy'schen Krystallen auch $\{210\}=\{11 \overline{2} 3\}$; diese Form konnte ich an dem mir zur Verfügung stehenden Material nicht beobachten.

Als sichere neue Formen kommen also zu den in Dana's Handbuch und Appendix aufgezählten Formen folgende drei:

$$
\begin{aligned}
\{4 \overline{1} T\} & =\{50 \overline{5} 2\} \frac{5}{2} R \\
\{11.5 .5\} & =\{20 \overline{2} 7\}_{\overline{7}} R \\
\{13.43 .8\} & =\{07 \overline{7} 6\}-\frac{7}{6} R,
\end{aligned}
$$

und als einigermassen wahrscheinlich die auf vor. S. zusammengestellten.

\section{Zwillinge.}

Zwillingslamellen nach dem Grundrhomboëder kommen - wie schon erwähnt - in den birmaner Rubinkrystallen ziemlich häufig vor, Juxtapositionszwillinge nach demselben Gesetze habe ich jedoch nicht gesehen. (Einen solchen beschreibt und zeichnet z. B. Lasaulx $\left.{ }^{1}\right)$.) Viel seltener kommt an Korund die Basis als Zwillingsebene vor. Dieses Zwillings1) I. c. 
gesetz kann bekanntlich auch so ausgedrückt werden: Zwillingsebene das Prisma erster Art $\{2 \bar{T} T\}=\{10 \bar{T} 0\}$. Jeremejew erwähnt einen solchen unter russischen Krystallen ${ }^{1}$ ) und auch Bauer erwähnt und zeichnet einen birmaner Krystill'²), in welchem eine Jünne Zwillingslamelle nach der Basis steckt. Unter dem von mir untersuchten Material vertritt nur ein Krystall dieses /willingsgeselz, doch ist derselbe in einem Sextanten als regelmässiger Juxtapositionszwilling ausgebildet. In lig. 4, Taf. XIV habe ich ihn ergänzt gezeichnet. Zur Bekräftigung des Zwillingsgesetzes wurde an diesem Krystalle gemessen:

$$
r \underline{v}=(100):(100)=(10 T 1):(10 T 1)=64048^{\prime} \quad 1 \quad 64046^{\prime} 43^{\prime \prime}
$$

Unter den Frémy'schen Rubinen ist an den kleinen Krystallen keinerlei Art Zwillingsbildung zu beobachten. l)ie Täfelchen jedoch, von welchen die grössten $1 \mathrm{~cm}^{2}$ gross sind, sind Zwillinge nach demselben zweiten Gesetze, wie der vorhin erwähnte einzige birmaner Krystall, nur ist hier die Art der Verwachsung eine andere (Fig. 22, 23 und 24, Taf. XV). Diese Täfelchen fasst Des Cloizeauxis) als einfache Krystalle auf, indem er die einen Rhombö̈derflächen als $\eta\{22 T)=\{01 T 1\}$ nimmt. Dass hier keine einfachen Krystalle, sondern Zwillinge vorliegen, zeigt die feine Streifung auf der Basisfläche, welche wie an den birmaner Krystallen den Kanten or parallel geht. Die Verwachsung ist übrigens so fein, dass man auf der Basisfläche die /willingsgrenze nur hier und da und nur recht schwach beobachten kann.

\section{Brechungsindices.}

Methode. Unter den untersuchten birmaner Krystallen befinden sich mehrere prismatisch ausgebildete schöne Krystalle, welche zur Bestimmung der Brechungsindices so zu sagen einladen, und auch die ceyloner und dic Frémy'schen Krystalle zeigten sich dazu geeignet. Ich halse die Gelegenheit benutzt, um so mehr als über die Brechungsexponenten des Korund bisher sehr wenig Daten vorhanden sind, und zwar einige von Des Cloizeaux ${ }^{4}$ ) und eine von $\left(0 \operatorname{san}{ }^{5}\right)$ :

$\begin{array}{lcccc} & \text { Lichtart: } & \omega & \varepsilon & \text { Beob.: } \\ \text { Hellblauer Korund } & \text { Roth } & 1,767-1,768 & 1,759-1,760 & \text { Dx. } \\ \text { Rubin } & \text { Roth } & 1,768 & 1,759 & \text { 1)x. } \\ \text { Korund von Ceylon } & D & 1,7690 & 1,7598 & \text { Os. }\end{array}$

Es gab ferner die verschiedene Färbung der Krystalle Gelegenheit zu erforschen, ob zwischen Färbung und den Brechungsverhältnissen ein Z $/ u$ -

1) Ref. diese Zeitschr. 3, 438.

2) l. c. S. 211 , Fig. A. 3) l. c.

4) Ann. des mines $1858,14,354$.

5) Rosenbusch, Mikr. Physiogr. 1892, 385 . 
sammenhang existire oder nicht. Um diesbezüglich ein Resultat zu erzielen, galt es zunächst festzustellen, inwiefern die Lichtbrechungsverhältnisse bei gleichgefärbten Krystallen variiren; ich habe deshalb die Bestimmungen nicht an einigen, sondern an einer grösseren Anzahl Krystalle gemacht.

Als Methode diente die Beobachtung der minimalen Deviation. Für diejenigen Krystalle (Nr. 4, Nr. 2 und die Frémy'schen), bei welchen das brechende Prisma eine Basisfläche und eine entgegenliegende Rhomboëderfläche bildeten, habe ich zur Berechnung von $\varepsilon$ die von Liebisch mitgeteilten Hormeln $\left.{ }^{1}\right)$ Jenutzt:

$$
\begin{aligned}
& A^{0} \mathrm{e}^{4}+2 A_{1} \mathrm{e}^{2}+A_{2}=0 \\
& A^{0}=\sin ^{2} \tau \\
& 2 A_{1}=\left(\left(\mathrm{o}^{2}+\frac{\sin ^{2} \varrho}{C^{2}}+\frac{\cos ^{2} \varrho}{S^{2}}\right) \cos ^{2} \tau-\frac{1}{C^{2}}-\frac{1}{S^{2}}\right. \\
& A_{2}=\mathrm{o}^{2}\left(\frac{\sin ^{2} \varrho}{C^{2}}+\frac{\cos ^{2} \varrho}{S^{2}}\right) \cos ^{2} \tau+\frac{1}{C^{2} S^{2}} \\
& 1_{C}=\frac{\cos \frac{A}{2}}{\cos \frac{A+\delta_{e}}{2}}, \frac{1}{S}=\frac{\sin \frac{A}{2}}{\sin \frac{A+\delta_{e}}{2}},
\end{aligned}
$$

in welchen im vorliegenden Falle

$$
\begin{aligned}
\varrho & =90^{\circ}-\frac{A}{2} \\
\tau & =0 .
\end{aligned}
$$

Als Refractometer diente in München das Fuess'sche grosse Goniometer ( $\mathrm{Nr}$. I), in Budapest ein gewöhnliches (Nr. II) von derselben Firma ${ }^{2}$ ). Ich habe beide vor dem Gebrauche rectificirt.

1) Physik. Krystallogr. S. 389.

2) Ich kann nicht umbin, bei dieser Gelegenheit moine Heinung über die Verwendbarkeit dieser beiden Instrumente als Spectrometer auszusprechen. Nach meinen Erfahrungen entspricht weder das eine, noch das andere vollkommen diesem Zwecke. Der Vortheil des grossen Goniometers ist jedenfalls der grosse Limbus, scine genaue Eintheilung und die genaue und zugleich bequeme Ablesung (von 10", schätzung von $4^{\prime \prime}$, aber ein Nachtheil ist, dass die Fernröhrc, dem speciellen $Z$ wecke dieses Instrumentes entsprechend, weit von einander abstehen, so dass viel Licht verloren gelıt. Beim Goniometer $\mathrm{Nr}$. II ist umgekehrt die Genauigkeit der Ablesung keine befriedigende, denn man kann darauf nur $30^{\prime \prime}$ sicher ablesen; die schätzung von $15^{\prime \prime}$ wird durch die Fehler der Kreistheilung ganz illusorisch gemacht und hat nur dann Sinn, wenn man beide Nonien abliest, was oft sehr unbequem ist, oder die Messung mit einem anderen Theile des Limbus wiederholt resp). das Repetitionsverfahren anwendet. Im vorliegenden Falle - der brechende Winkel war bei allen Krystallen gross - war die Ablesung zwar genügend (ich wiederholte jede Messung mit einem oder zwei anderen Theilen des Limbus), aber es wäre doch wünschenswerth, dass diese Goniometer: 
Um dic Bestimmungen in möglichst mehr und zugleich intensiven Lichtarten zu vollführen, hatte Prof. Krenner die Güte, auf meine Bitte den Wülfing'schen kleineren Spectralapparat anzuschaffen, welcher bekanntlich ein Attribut zu den Goniometern Nr. II bildet und dessen Brauchbarkeit durch die Untersuchungen des Autors über die Brechungsexponenten des Eisenglanz und des Turmalins genügend bekannt ist. Leider konnte ich wegen der ungünstigen Jahreszeit diesen Apparat nicht mit Sonnenlicht benutzen, für welches or eigentlich construirt ist, sondern nur mit künstlichem Lichte, und zwar stand mir meist nur gewölnnliches Auerlicht zur Verfügung. Ich suchte also vorerst festzustellen, wie weit die liesultate unter solchen Umständen genau seien. Bei Auerlicht muss man natïrlich den Collimatorspalt des Instrumentes etwas weiter iflinen, als es für die Reinheit des entstehenden Spectrums zweckmässig ist, und auch den Wels sky-Spalt etwas besser offnen $\left(1 \frac{1}{2}-2^{\prime}\right)$, so dass also das herausgeschnittene Mittelstück des Spectrums viel weniger monochromatisch ist, als mit Sonnenlicht. Ein weiterer Uebelstand ist, dass wegen der Weite des Collimatorspaltes auch die Einstellung viel weniger genau ist (zur Einstellung habe ich nicht die eingewerthete Lintheilung der Schritube, sondern $\mathrm{Na}$-, $\mathrm{Tl}$-Licht und die $\mathrm{H}$-Linien benutzt), als die Einstellung der l'raunhofer'schen Linien.

Es ist aus Obigem klar, dass man sich also bei Benutzung von Auerlicht mit einer minderen Genauigkeit begnügen muss, als mit Sonnenlicht, und zwar betrügt diese nach meinen Messungen an einem Quarzkrystalle und mehreren Rubinkrystallen - also bei grossem brechenden Winkel durchschnittlich $\pm 0,0005$. Reicht dies, dann leistet dieser $\Lambda$ pparat auch mit gewöhnlichen Auerlicht vorzügliche Dienste, und ich bin überzeugt, dass man mit Zirkonlicht oder starkem elektrischem Lichte ebenso die übliche Genauigkeit $( \pm 0,0002)$ erzielen kann, als mit Sonnenlicht, nur muss man auch dann zur Einstellung nicht die Schraube, sondern die erwähnten Lichtarten $(\mathrm{Na}-, \mathrm{Tl}-, \mathrm{H}$-Linien) benutzen.

Nach diesen Erfahrungen nahm ich - meinem Zwecke entsprechend wieder zum $\mathrm{Na}$-Licht und zu den Geissler'schen Röhren Zuflucht. Um müglichst intensives monochromatisches Licht zu haben (das erforderte die einigermassen starke Absorption der dunkleren Krystalle!, mischte ich $\mathrm{NaCl}$

welche als solche von den mebrkreisigen Instrumenten mit der Zeit ohnedies verdrängt werden, einen etwas grösseren und feiner getheilten Limbus hätten, damit sie als Refractometer für alle Fälle benutzt werden könnten, sowie für feinere goniometrische Messungen (Vicinalflächen, Ablenkung einzelner Fläcben, sehr kleine Winkel bei Zwillingskrystallen). Ihre Optik ist für diese $Z$ wecke ganz gut (ich habe bei vorliegender Lintersuchung immer das Ocular $\beta$ gebraucht). Wünschenswerth wäre noch, dass der Websky'sclıe Spalt, welcher sich für spectrometrische Messungen so vortheilhaft bewährt hat, auf die Art der spectrometrischen geraden Spalte zu ölfnen ware, so dass er immer ein bisymmetrisches Bild lieferte. 
mit $\left.\mathrm{Na}_{2} \mathrm{SO}_{4}{ }^{1}\right)$ und stellte dic Laspeyres'sche Lampe nicht quer, sondern liings dem Collimatorrohre 2); von Geissler'schen Röhren benutzte ich die knieförmig gebogenen Riedel'schen ${ }^{3)}$, welche sich als bedeutend lichtstärker crwiesen, als gewöhnliche Röhren. Nur bei zwei dunkelrothen Krystallen (Nr. 4 und Nr. 2) konnten die abgelenkten Reflexe in Folge der starken Absorption für $\mathrm{H}_{3}$ nicht eingestellt wcrden.

Ich habe bei Mcssung der minimalen Deviation nicht $2 \delta$ sondern $\delta$ abgelesen, so dass ich beide Strahlen für cine Farbe wenigstens dreimal einstellte und wenigstens zwcimal gerade auf den Spalt. Bei der letzten Finstellung auf den Spalt wurde der Krystall immer entfernt. Diese gerade Einstellung auf den Spalt hat keine Schwierigkeiten, wenn das Fadenkreuz und der Spalt genau justirt sind und wenn man die Stärke der Lichtquelle entsprechend vermindert, z. B. dadurch, dass man vor die $\mathrm{Na}$-Flamme cin weisses oder gelbliches, vor die Geissler'sche Röhre (ganz an sie heran) rothes Seidenpapier bringt.

Da zur Messung immer natürlichc Krystallflächen dienten (nur bei Krystall Nr. 2 musste die eine Iläche durch Anschleifen und Poliren verbessert werden), habe ich bei jedem Krystalle darauf geachtet, dass sich der brechende Winkel und dic Deviationen auf cin und dieselbe kleine Flächenpartie beziehen, indem ich die übrigen mit matter schwarzer Farbe abblendete.

Messungsresultate. Die Mittel der Messungen und die daraus berechneten Brechungsexponenten theile ich im Folgenden mit. Jeder Werth für die brechenden Winkel beruht also wenigstens auf sechs, und für die Deviationen auf fünf Linstellungen auf je zwei verschiedenen Theilen des Limbus. Für die Krystalle Nr. 2 und 4, sowie für die Frémy'schen Krystalle hatte Herr Dr. $\boldsymbol{A}$. Anderko, Adjunkt des k. ung. meteorol. Institutes, die Güte die Brechungsexponenten $\varepsilon$ zur Controlle auch auszurechnen, wofür ich ihm auch an dieser Stelle besten Dank sage. (In folgender Tabelle bedeuten die oberen Daten den brechenden Winkel.)

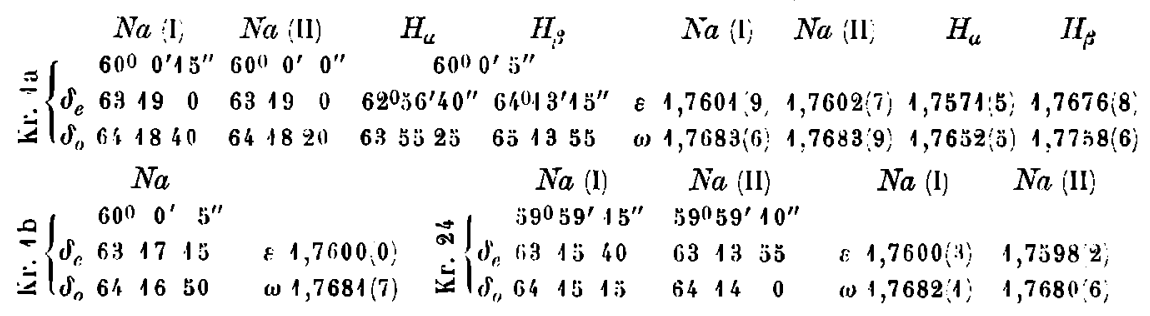

1) Diese Mischung bat sich sehr gut bewährt. Noch intensiver ist $\mathrm{NaBr}$, jedoch muss man bei seiner Benulzung einen gut ziehenden $A$ bschluss haben.

2) Meiner Ansicht nach sollte dieser, Physikern wohlbekannte Handgriff zur Erhöhung der Lichtstärke in den krystallogr. Lehrbiichern berücksichtigt werden.

3) Zeiss'scher Specialkatalog für Spectrometer uud Refractometer 1999, S. 5. 


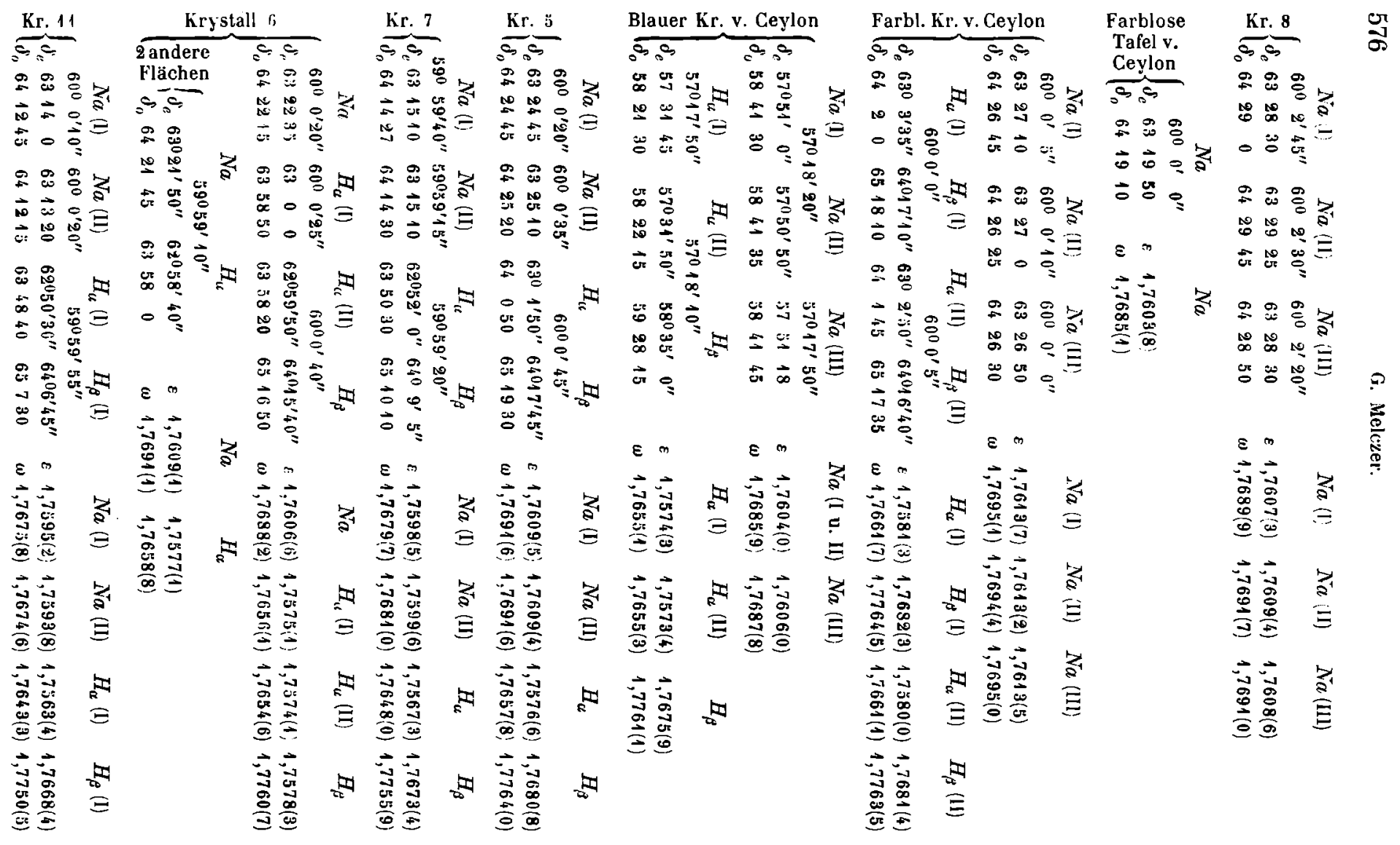



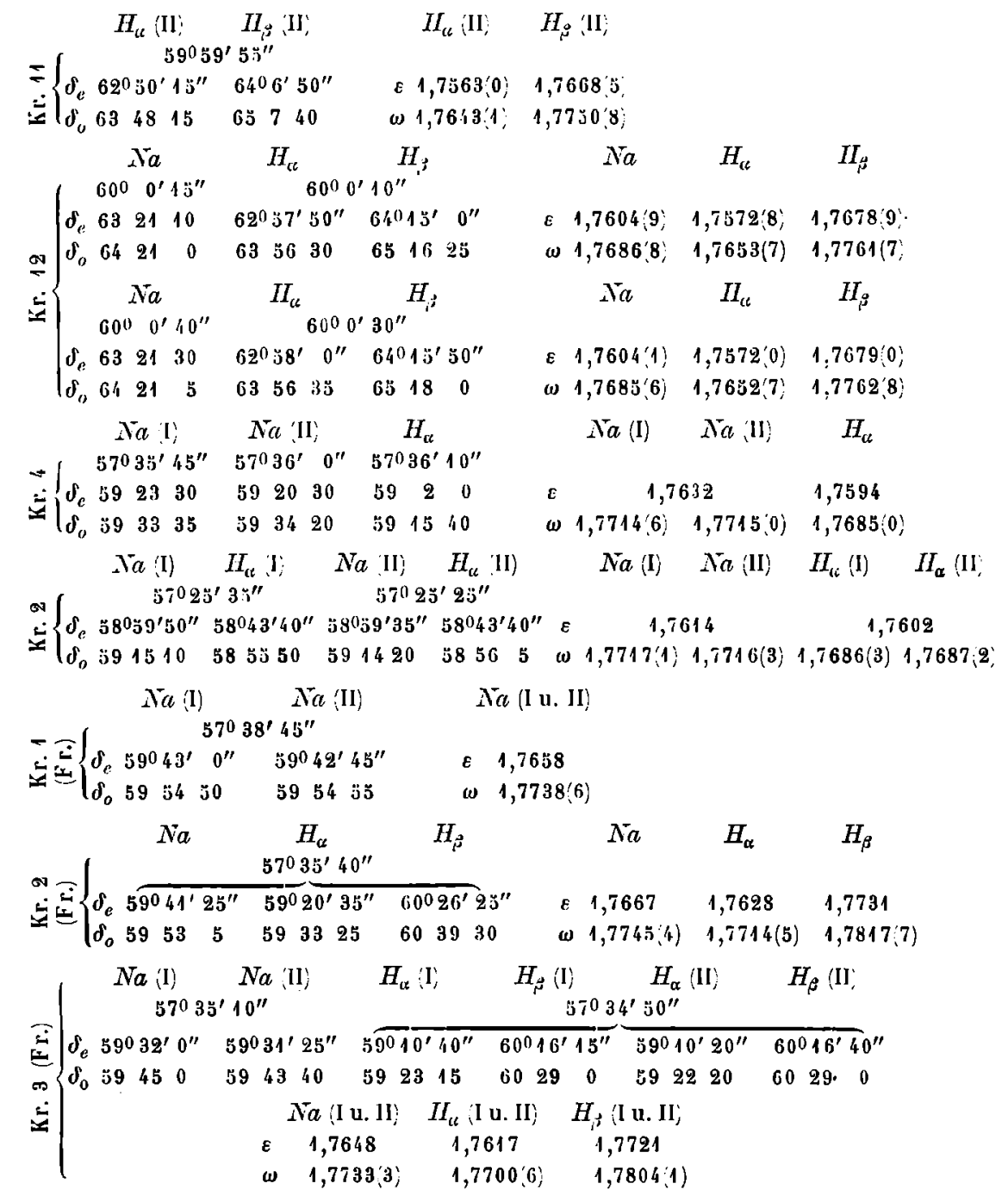

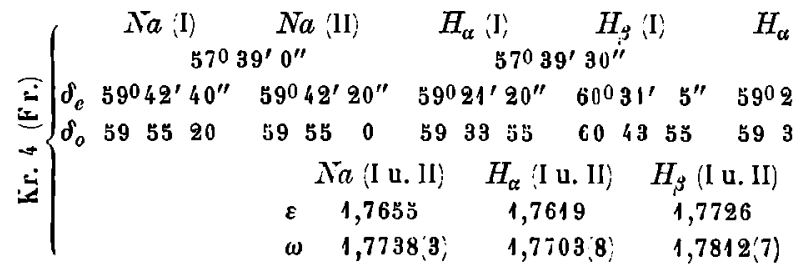

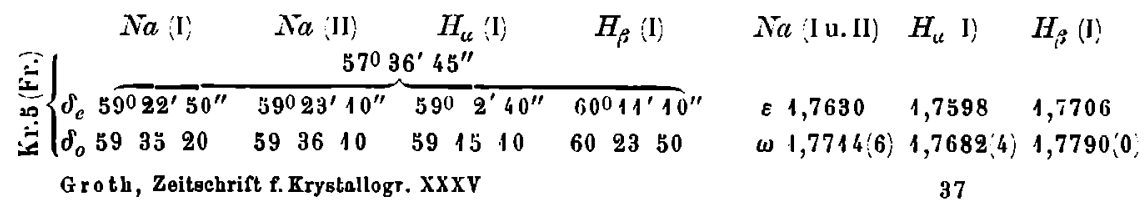




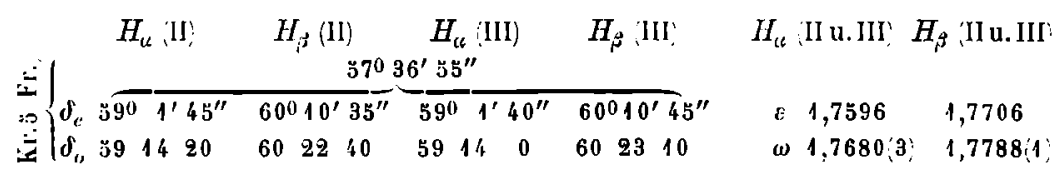

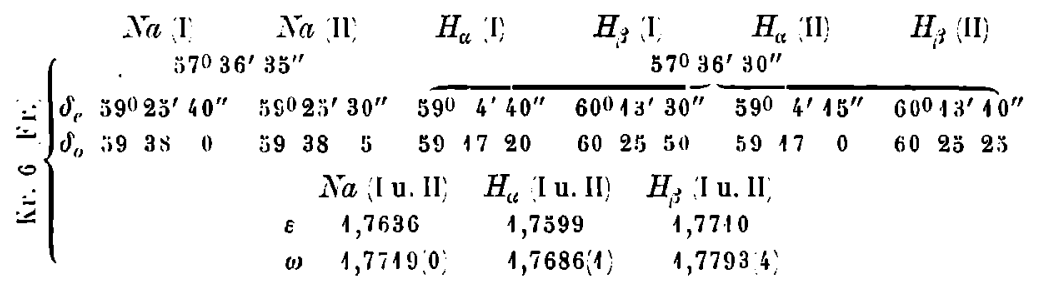

Bei der Zusammenstellung der Messungsresultate (siehe folgende Tabelle) stellte es sich heraus, dass zwischen der Intensität der rothen Färbung und zwischen der Lichtbrechung ein ausgesprochener Zusammenhang existire. Unter den gemessenen Rubinen ist nämlich Krystall Nr. 11 auffallend licht gefärbt, nach ihm folgt in dieser Beziehung Nr. 7, andererseits sind Krystall 4 und 2 - wie schon erwähnt die dunkelsten - recht schön rubinroth; die Lichtbrechung letzterer ist erheblich grösser und erreicht die der Frémy'schen Krystalle, welche dunkler sind als die birmaner Krystalle. Die übrigen Krystalle haben ungefähr dieselbe rothe Färbung, jedoch kann man bei aufmerksamer Betrachtung sehen, dass Krystall 6, 8 und 5 etwas dunkler sind als 12, 1a, $1 \mathrm{~b}$ und 24. Die farblosen Krystalle und der blaue Krystall nehmen in der Reihenfolge nicht Endstellen ein, wie man vielleicht erwarten müchte, sondern stehen so ziemlich in der Mitte.

In der Tabelle sind die Brechungsexponenten, sowie die aus ihnen berechneten Werthe für Dispersion und Doppelbrechung enthalten, sämmtlich auf vier Decimalen abgerundet. Diejenigen Krystalle, für welche nur Bestimmungen für $\mathrm{Na}$-Licht vorliegen, gaben keine guten Reflexe, so dass ich sie in Roth und Blau nicht bestimmte. Die auf sie bezüglichen, sowie für Krystall Nr. 4 und 2 und den Frémy'schen Krystall Nr. 2 mitgetheilten Werthe sind nur bis auf $\pm 0,0003$ bis 0,0004 , die übrigen auf $\pm 0,0002$ bis 0,0001 genau. Ganz falsch ist $\varepsilon_{D}$ für Krystall Nr. 2; der Fehler steckt, wie ich mich überzeugt habe, nicht in der Berechnung.

(Hierher nebenstehende Tabelle.;

Aus der Tabelle ist ferner sichtbar, dass trotz den grossen Unterschieden in der Lichtbrechung die Dispersion sowohl für den ordinären, als auch für den extraordinären Strahl nahezu gleich ist $\left\langle\omega_{D-F}\right.$ und $\varepsilon_{D-F}$ ist bei den lichter gefürbten etwas grüsser, als bei den dunkler gefürbten, $\varepsilon_{c-D}$ umgekehrt). Bei den natürlichen Krystallen ist in der Doppelbrechung kein Unterschied zwischen lichter und dunkler gefärbten Krystallen, wohl aber ein Unterschied zwischen den natürlichen und künstlichen Krystallen. Bei den letzteren ist nämlich $\omega_{C}-\varepsilon_{C}$ und $\omega_{D}-\ddot{c}_{D}$ grüsser, als 


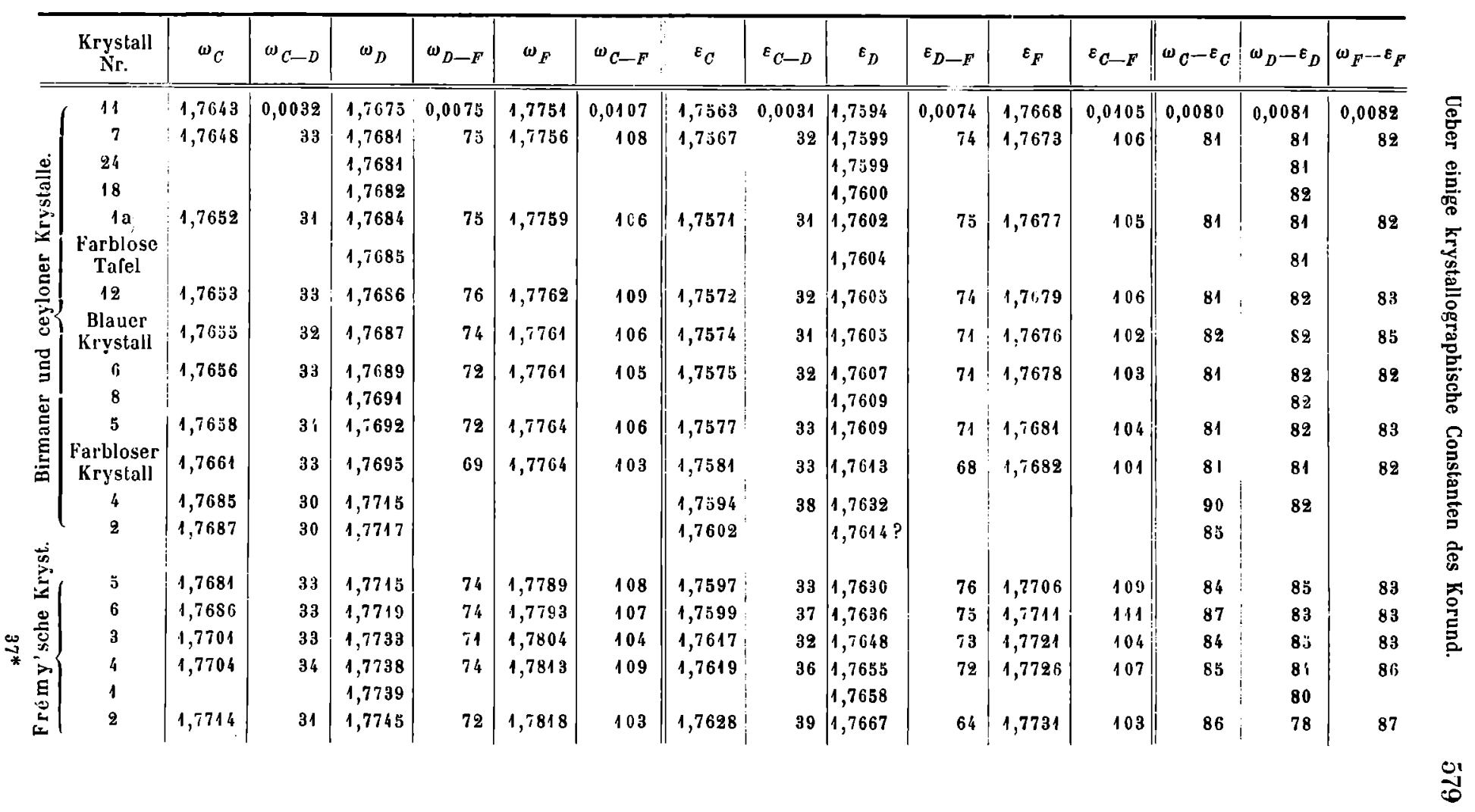


bei den natürlichen und $\omega_{C}-\varepsilon_{C}>\omega_{F}-\varepsilon_{F}$, während bei den natürlichen $\omega_{F}-\varepsilon_{F}>\omega_{C}-\varepsilon_{C}$.

Wie aus der Tabelle ersichtlich, ist für petrographische Zwecke anstatt des bisher angenommenen Werthes $\left(v-\varepsilon=0,009^{1}\right)(u-\varepsilon=0,008 \mathrm{zu}$ setzen.

Um zu unterscheiden, ob der grosse Unterschied in der Lichtbrechung nur der relativen Menge eines diluten Farbstoffes zuzuschreiben sei, oder ob beide eine Folge verschiedener chemischer Zusammensetzung seien, habe ich erstens die zwei lichtesten und die zwei dunkelsten (Nr. 2 und 4) Krystalle von Neuem geometrisch gemessen, ob eventuell auch in dem Axenverhältnisse derselben ein Unterschied sei, zweitens hatte auf meine Bitte Herr Chemiker J. Loczka, Custos des ung. Nationalmuseums, die Güte, einige Bestimmungen des spec. Gewichtes auszuführen.

Die Messung der erwähnten Krystalle ergab:

\begin{tabular}{|c|c|c|c|}
\hline Krystall Nr. 11 & $c r=57^{0} 36^{\prime} 50^{\prime \prime}$ & $n$ & $\begin{array}{l} \pm d \\
20^{\prime \prime}\end{array}$ \\
\hline (licht) & $r r=93 \quad 5920$ & 2 & 40 \\
\hline $\begin{array}{c}\text { Krystall Nr. } 7 \\
\text { (licht) }\end{array}$ & 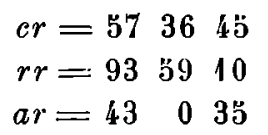 & $\begin{array}{l}3 \\
2 \\
6\end{array}$ & $\begin{array}{r}1 \\
1 \\
10 \\
30\end{array}$ \\
\hline $\begin{array}{l}\text { Krystall Nr. } 4 \\
\text { (dunkel) }\end{array}$ & $\begin{array}{rrr}c r=57 & 36 & 20 \\
r r=93 & 59 & 59\end{array}$ & $\begin{array}{l}6 \\
4\end{array}$ & $\begin{array}{l}30 \\
40\end{array}$ \\
\hline $\begin{array}{l}\text { Krystall Nr. } 2 \\
\text { (dunkel) }\end{array}$ & 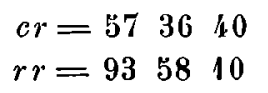 & $\begin{array}{l}4 \\
2\end{array}$ & $\begin{array}{r}150 \\
20\end{array}$ \\
\hline
\end{tabular}

In Anbetracht der Differenzen $( \pm d)$ und dessen, dass das Mittel für or bei den ebenfalls dunkel gefürbten Frémy'schen Krystallen (s. S. 567) ebenfalls $57^{\circ} 36^{\prime} 40^{\prime \prime}$ ist, kann man also einen Unterschied zwischen dem Axenverhältnisse der lichten und der dunkleren Rubine nicht constatiren.

Zur Bestimmung des spec. Gewichtes dienten zwei Krystalle, der schon gemessene dunkle (Nr. 2) und ein zweiter lichter. Am letzteren bekam ich:

$$
\begin{aligned}
& 57032^{\prime} 13^{\prime \prime} \pm 10^{\prime \prime} \\
& \delta_{0} 59540 \pm 20, \text { also } \\
& \omega_{N a}=1,7681 ;
\end{aligned}
$$

seine Lichtbrechung war also gleich des sehr licht gefärbten Krystalles Nr. 7.

Das Resultat ist nach der gütigen Mitwirkung des Herrn Loczka folgendes:

1) Rosenbusch, Mikr. Phys. (3. Aull.:, 1, 166 und Michel Lévy et Lacroix, Les Min. des roches p. 97. 
Spec. Gew.:

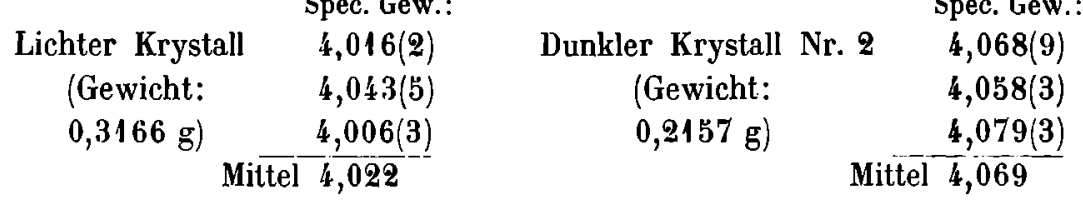

Da diese zwei Krystalle - wie auch im Allgemeinen die meisten vollkommen durchsichtig sind und kleine Einschlüsse oder andere Inhomogenität in ihnen bemerkbar ist, muss der Unterschied im spec. Gewicht wohl auf einem quantitativen Unterschiede in der chemischen Zusammensetzung beruhen, welcher wahrscheinlich auch die Ursache der Unterschiede in der Lichtbrechung ist.

Zur Entscheidung dieser Frage wird vielleicht die chemische Analyse dieser beiden Krystalle beitragen, welche im Gange ist und über deren Resultat später berichtet wird. 